\title{
Effectiveness of telenutrition in a women's weight loss program
}

Isaac E Kuzmar, Ernesto Cortés-Castell, Mercedes Rizo

Objective: The objective of this study is to evaluate the effectiveness of telenutrition versus traditional nutritional consultation for female obese patients that needs nutritional treatment. Methods: A comparative clinical study was conducted among 233 (including 20 dropouts and 60 failures) obese or overweight women who consulted a nutrition clinic in Barranquilla (Colombia) for nutritional assessment choosing telenutrition or traditional attention that includes a weekly follow-up consultation over 16 weeks, food consumption patterns, Body Mass Index $\left(\mathrm{BMI}, \mathrm{kg} / \mathrm{m}^{2}\right)$ register, waist and hip circumference register. Treatment response and difference between telenutrition and traditional consultation were made according to BMI, waist, hip and initial-waist/height ratio, calculating the relative risk. Results: In 68 (29.2\%) women who chose traditional attention, 9 (37.5\%) dropped out, 24 (40\%) failed and 35 (23.5\%) were successful, showing 1.4\%(1.0 SD) BMI loss, 5.8\% (3.4 SD) in waist circumference, $4.5 \%$ (2.8 SD) in hip circumference and $0.04 \%$ (0.02 SD) in iwaist/height ratio. In 165 (70.8\%) women who chose telenutrition, 15 (62.5\%) dropped out, 36 (60\%) failed and 114 (76.5\%) were successful, showing 1.1\% (1.0 SD) BMI loss, $5.0 \%$ (3.2 SD) in waist circumference, 3.5\% (3.1 SD) in hip circumference and $0.03 \%(0.02$ $\mathrm{SD}$ ) in iwaist/height ratio. A significance level of $\mathrm{p}<0.05$ is considered. Conclusion: Telenutrition has a failure or dropout risk factor about half values of traditional consultation with slightly statistically significant differences. This study concludes that telenutrition can support or sometimes replace the traditional consultation when developing weight loss programs in obese women. 


\section{Title: EFFECTIVENESS OF TELENUTRITION IN A WOMEN'S WEIGHT \\ 2 LOSS PROGRAM}

3
4
5
6

7 Email:

8

9

10

11

12

13

14

15

16

17 Email:

Email:
Isaac Kuzmar

Department of Nursing

Faculty of Health Sciences

University of Alicante, Alicante, Spain

isaackuzmar@yahoo.es

\section{Ernesto Cortés-Castell}

Department of Pharmacology, Pediatrics and Organic Chemistry School of Medicine

Miguel Hernández University, Alicante, Spain

ernestocort@gmail.com

\section{Mercedes Rizo}

Department of Nursing

Faculty oh Health Sciences

University of Alicante, Alicante, Spain

mercedes.rizo@ua.es
18

19

20

21

22

23

24 Email:
Isaac Kuzmar

Department of Nursing

Faculty of Health Sciences

University of Alicante, Alicante, Spain

Carretera de San Vicente del Raspeig, s/n, 03690

Sant Vicent del Raspeig, Alicante, Spain

isaackuzmar@yahoo.es 


\section{INTRODUCTION}

26 The solutions in home care, is becoming a response to the need to control health care costs of the 27 population. Advances in information and communication technology (ICT) have directly 28 influenced the development of Telemedicine and Telecare solutions ${ }^{1}$.

29 Besides the change in the form of providing medical care, telemedicine is becoming an industry 30 that can generate multiple billions of dollars, so there is a need to create successful programs that 31 provide clinical services remain profitable ${ }^{2}$.

32 The application of telehealth principles by registered dieticians or doctors to deliver medical 33 nutrition therapy is termed telenutrition ${ }^{3}$.

34 Obesity is a multifactorial disorder related to genetic background, environmental factors, 35 behavioural, underlying diseases and socioeconomic status ${ }^{4}$. It is known that the prevalence of 36 obesity has experienced an alarming worldwide increase ${ }^{5}$; therefore, we can say that we are in the 37 midst of a global obesity epidemy ${ }^{6}$. The physiology of obesity is based on an imbalance between 38 caloric intake and energy expenditure ${ }^{7}$. Obesity has become a very frequent source of medical, 39 nursing or nutrition advice 8 .

40 The status of overweight and obesity has connotations relating to the patient's own body image. 41 The appearance or perception of body image is defined as body shape that is made by the mind 42 plus the fact that consists of subjective representations of physical appearance 9 . The concept of 43 body image varies throughout life, depending on the social influences and life situations that 44 affect the behavior ${ }^{10}$. Dissatisfaction with physical appearance related to body weight is higher in 45 women than men varying across ethnic groups related to the cultural integration degree ${ }^{11}$. Some 46 authors find no relationship between dissatisfaction with body appearance and body mass index 47 (BMI) in obese and overweight women ${ }^{12}$.

48 49

Obesity has more adverse effects in women than men, in relation to cardiovascular risk; in turn, some health professionals are reluctant to initiate treatment of comorbidities of obesity and metabolic syndrome in women, because they perceive less risk, than that seen in men ${ }^{13}$. 
51 There are several studies showing that an overweight and obesity treatment to be performed for a

52 few months, more women than men attend, but leave $42 \%$ for various reasons, including a lack of

53 motivation with the results of weight loss and the economic cost ${ }^{14}$ and it is demonstrated that

54 social classes, level of education, marital status, alcohol and tobacco consumption are not

55 regarded as influential factors in the successful outcome of treatment in overweight and obese 56 patients $^{10}$.

57 Is necessary to give to all nutrition consultations a global approach, that adds to the necessary 58 changes in eating habits, exercise and other respects in accordance with the acquired body image, 59 health, etc. producing an adherence to the acquired new habits. Socioeconomic and demographic 60 changes are occurring very rapidly in some areas of the world, accompanied by changes in 61 lifestyle, dietary patterns and the epidemiological profile of prevalent diseases ${ }^{15}$.

62 Telehealth network can serve as a model for integrating health services ${ }^{16}$ including telenutrition ${ }^{2}$. 63 Commercial industries have outpaced traditional healthcare consultation in terms of traditional 64 approaches to weight control for electronic online delivery. Little is known about the 65 effectiveness of telenutrition. This gap represents a barrier to developing successful, patient66 based e-health applications for effectiveness behaviour change. The main objective of this paper 67 is to determine the effectiveness of telenutrition versus a traditional healthcare consultation in a 68 weight loss program.

\section{MATERIAL AND METHODS}

70 Subjects

71 A clinical intervention and e-health study was conducted among 233 (according to the WHO 72 classification ${ }^{17}$ ) overweight and obese women who consulted a nutrition clinic in Barranquilla 73 (Colombia) for the purpose of nutritional assessment by telenutrition and traditional in-person 74 consultation. They were subject to a personalized weekly follow-up consultation over the course 75 of 16 weeks in which food consumption patterns, and measures were registered. The inclusion 76 criteria were female gender, voluntary assistance, the use of internet communications, not 77 excluding those with chronic diseases such as kidney failure, cancers, hypertension, diabetes and 78 dyslipidemia, etc. that requires also medical follow up. This study also considers patients who 79 tried to lose weight in the previous month or earlier. Exclusion criteria were male gender, and 
80 those who not qualify for inclusion criteria. In turn, alcohol or tobacco consumption does not

81 affect actual results. The study was conducted according to Helsinki's rules obtaining all patients

82 informed consent.

83 Methods

84 Weight loss results in patients could be seen in 16 weeks ${ }^{5}$. Women were asked to choose the 85 treatment to be followed by telenutrition or traditional consultation. In both groups (telenutrition 86 and traditional consultation), the study included an initial in-person consultation with a complete 87 medical record (date accessed, date of birth, personal identification data including e-mail and 88 messenger service, socioeconomic status, educational level, personal medical history, toxic 89 precedents, etc.) and depending on the group, a virtual or physical weekly WHO recommended 90 nutritional assessment ${ }^{18}$ (age, height and weight, waist and hip perimeter). With an eating habits 91 questionnaire $^{19}$ response we made the weekly WHO-based ${ }^{20}$ low calorie diets. Patients who 92 followed telenutrition were instructed and taught on first in-person consultation on how to 93 measure and read waist, hip, and weight scale. Nutritional assessment and diet were sent by 94 email and the patient controls by online internet messenger. In week number sixteen, 95 telenutrition patients were also evaluated in physical consultation to control the results. 96 Telenutrition patients were weekly controlled and supervised by email, messenger and computer 97 chat, and those who chose traditional consultation by in-person weekly nutritional assessment at 98 the clinic.

99 The failure criteria used is when the patient did not lose weight or did not lose measures; and the 100 success criteria is when the patient loses weight or loses measures after 16 continuous weeks of 101 monitoring either by telenutrition or traditional consultation.

102 With the obtained data we calculate the initial and final BMI according to WHO criteria ${ }^{17}$ as well 103 as weight, waist, hip, initial and final waist/height ratio loss percentages. The data were treated 104 using IBM SPSS Statistics version 22.0 software, checking the relative risk. A significance level 105 of $\mathrm{p}<0.05$ is considered using U Mann-Whitney. This study was approved by the institution 106 SEMI-Servicios Médicos Integrados of Barranquilla, Colombia.

\section{RESULTS}


108 A total of 233 women were interviewed, of whom 68 (29.2\%) chose traditional consultation and $109165(70.8 \%)$ chose telenutrition. $24(15(62.5 \%)$ telenutrition versus $9(37.5 \%)$ traditional) 110 patients dropped out the study with no known reasons, representing 89.7\% (209) follow-up

111 tracking; we assumed that patient discontinued the treatment because they were unmotivated.

112 Our focus groups included 233 individuals with 165 (70.8\%) in the telenutrition group and 68 $113(29.2 \%)$ in the traditional healthcare consultation group (Figure 1).

$11436(60.0 \%)$ patients who followed telenutrition versus $24(40.0 \%)$ patients who followed the 115 traditional consultation did not drop weight, considering them to be failures and $114(76.5 \%)$ 116 patients who followed telenutrition versus 35 (23.5\%) patients who followed traditional 117 consultation have lost weight (Figure 1). Significant differences were found in relation to 118 comparative results (Table 2).

119 Table 1 gives descriptive information. As indicated previously, the objective of the study is to 120 evaluate the effectiveness of telenutrition versus traditional nutritional consultation for obese 121 patients measuring their weight, waist and hip loss results.

122 Responses from our focus group participants suggest that most patients chose telenutrition for 123 their overweight treatment. Telenutrition gives home based support to some participants using 124 ICT, the results are quite similar.

125 Table 2 shows BMI, hip, waist, and iwaist/height loss percentage in the 149 patients who have 126 had success with traditional treatment showing 1.4\%(1.0 SD) BMI loss, 5.8\% (3.4 SD) in waist 127 circumference, $4.5 \%$ (2.8 SD) in hip circumference and $0.04 \%(0.02 \mathrm{SD})$ in $i$ waist/height ratio; 128 and in women who chose telenutrition showing 1.1\% (1.0 SD) BMI loss, 5.0\% (3.2 SD) in waist 129 circumference, $3.5 \%$ (3.1 SD) in hip circumference and $0.03 \%(0.02 \mathrm{SD})$ in $i$ waist/height ratio. 130 There is a slightly BMI\% $(\mathrm{p}=0.046)$ and Hip\% $(\mathrm{p}=0.040)$ difference in the traditional 131 consultation versus telenutrition. No statistical differences were found in waist $\%(\mathrm{p}=0.177)$ and 132 iwaist/height $\%(\mathrm{p}=0.131)$.

\section{DISCUSSION}


134 Prevalence of obesity is especially concerning because of its association with increased risk for

135 chronic health conditions risk which can be mitigated with weight loss ${ }^{21}$. Weight-loss programs

136 include education and support for improving dietary self-monitoring and behaviour change skills

137 in patients increasing social support ${ }^{22}$.

138 Alternative overweight and obesity treatments are very popular and despite being widely used, 139 have not been shown to be safe and effective ${ }^{23}$. Success weight loss rates are highly variable in 140 the literature ${ }^{24}$ and depend on many factors ${ }^{25}$. No scientific data of the studied population have 141 been found that can be used to compare to this research, making this study a pioneer in the 142 region.

143 The present study has used a comparative non-invasive clinical approach and has searched if 144 telenutrition can be more effective than the traditional healthcare consultation as determinants in 145 the success of a treatment for obesity in a Caribbean population. Patients who completed 146 treatment and therefore have lost weight in the week number 16 are $63.9 \%$ of the total that began

147 the treatment, despite the great difficulties that arise in the treatment of overweight and obesity.

148 The main limitation is that telenutrition was not allocated randomly and because of the data this 149 study did not use multivariate statistical analysis. The dropout rate is small (10.3\%) maybe 150 because the initial attendance is voluntary and for aesthetic reasons without a clinical condition or 151 maybe due to a lack of motivation.

152 It will be interesting to know if these results are similar in other world population regarding the 153 limitations; the authors are planning to make future research to complete this study.

\section{CONCLUSION}

155 Despite that telenutrition was not allocated randomly and not multivariate statistical analysis was 156 used, patients who chose telenutrition has a failure or dropout risk factor about half values of 157 traditional consultation with slightly statistically significant differences. Based on these results, 158 ICT-based using telenutrition can support or sometimes replace the traditional consultation when 159 developing weight loss programs in obese women. 


\section{REFERENCES}

161 1. While A, Dewsbury G. 2011. Nursing and information and communication technology (ICT): a discussion of trends and future directions. International journal of nursing studies 48(10): 1302-1310.

2. Kuzmar I, Rizo M, Cortés E. 2014. How to create a telemedicine service: telemedicine heptagon. Actual. Med. 99(791): 44-45

166

3. Chung LMY, Chung JWY. 2010. Tele-dietetics with food images as dietary intake record in nutrition assessment. Telemedicine and e-Health 16(6): 691-698.

4. Shea J, Diamandis EP, Sharma AM, Després JP, Ezzat S, Greenway F. 2012. The obesity epidemic. Clinical chemistry 58(6): 968-73.

5. Kuzmar I, Rizo M, Cortés-Castell E. 2014. Adherence to an overweight and obesity treatment: how to motivate a patient?. PeerJ 1-10. http://dx.doi.org/10.7717/peerj.495

6. Flegal KM, Carroll MD, Kit BK, Ogden CL. 2012. Prevalence of obesity and trends in the distribution of body mass index among US adults, 1999-2010. JAMA 307(5): 491-497.

7. Kuzmar I, Cortés E, Rizo M. 2014. Asistencia y respuesta al tratamiento por sobrepeso y obesidad en una población de Barranquilla (Colombia). Nutr. Clín. Diet. Hosp. 34(3): 18-26. Accepted for Publication

8. Macleod M, Gregor A, Barnett, C, Magee E, Thompson J, Anderson A. 2013. Provision of weight management advice for obese women during pregnancy: a survey of current practice and midwives' views on future approaches. Maternal \& child nutrition 9(4): 467-472.

9. Alwan H, Viswanathan B, Paccaud F, Bovet P. 2011. Is accurate perception of body image associated with appropriate weight-control behavior among adolescents of the Seychelles. Journal of obesity (2011)

10. Kuzmar I, Rizo M, Cortés E. 2014. Social classes, level of education, marital status, alcohol and tobacco consumption as predictors in a successful treatment of obesity. $J$ Nutr Disorders 4:1-3. http://dx.doi.org/10.4172/2161-0509.1000135

11. Fallon EA, Harris BS, Johnson P. 2014. Prevalence of body dissatisfaction among a United States adult sample. Eating behaviors 15(1): 151-158.

12. Cortese S, Falissard B, Pigaiani Y, Banzato C, Bogoni G, Pellegrino, M, Maffeis C. 2010. The relationship between body mass index and body size dissatisfaction in young adolescents: spline function analysis. Journal of the American Dietetic Association 110(7): 1098-1102. 
13. Mozaffarian D, Hao T, Rimm EB, Willett WC, Hu FB. 2011. Changes in diet and lifestyle and long-term weight gain in women and men. New England Journal of Medicine 364(25): 2392-404.

14. Carrasco F, Moreno M, Irribarra V, Rodríguez L, Martín M, Alarcón A, Mizón C, Echenique C, Saavedra V, Pizarra T, Atalah E. 2008. Evaluación de un programa piloto de intervención en adultos con sobrepeso u obesidad, en riesgo de diabetes. Rev Med Chile : 13-21.

15. Aballay R, Eynard A, Díaz M, Navarro A, Muñoz S. 2013. Overweight and obesity: a review of their relationship to metabolic syndrome, cardiovascular disease, and cancer in South America. Nutr Rev71(3): 168-179.

16. Dimmick S, Burgiss S, Robbins S, Black D, Jarnagin B, Anders M. 2003. Outcomes of an integrated yelehealth network demonstration project. Telemedicine Journal and eHealth 9(1): 13-23. doi:10.1089/153056203763317611.

17. WHO. 2013. Obesity and overweight. Fact sheet No.311. Available at http://www.who.int/mediacentre/factsheets/fs311/en/

18. WHO. 1995. Physical status: the use and interpretation of anthropometry. Technical report series 854: 312-411.

19. Dana-Farber Cancer Institute. Eating habits questionnaire. Available at: http://rtips.cancer.gov/rtips/viewProduct.do?viewMode=product\&productId=173387

20. WHO. 2014. Available at http://www.who.int.topics/diet/es/.

21. Diabetes Prevention Program Research Group. 2002. Reduction in the incidence of type 2 diabetes with lifestyle intervention or metformin. N Engl J Med 346: 393-403.

22. Burke LE, Wang J, Sevick MA. 2011. Self-Monitoring in Weight Loss: A Systematic Review of the Literature. J Am Diet Assoc. 111(1): 92-102

23. Allison DB, Fontaine KR, Heshka S, Mentore JL, Heymsfield SB. 2001 Alternative treatments for weight loss: a critical review. Crit Rev Food Sci Nutr 41: 1-28.

24. Hill AJ, Williams J. 1998. Psychological health in a non-clinical sample of obese women. Int J Obes 22(6): 578-83.

25. Paxton SJ, Wertheim EH, Gibbons K, Szmukler GI, Hillier L, Petrovich JL. 1991. Body image satisfaction, dieting beliefs, and weight loss behaviours in adolescent girls and boys. $J$ Youth Adolesc 20(3): 361-379. 


\section{1}

Overweight and obesity treatment success through traditional consultation and telenutrition.

The dropout or failure risk of telenutrition versus traditional consultation is $=0.45(95 \% \mathrm{Cl}$ :

$0.27-0.85 ; p<0.05)$

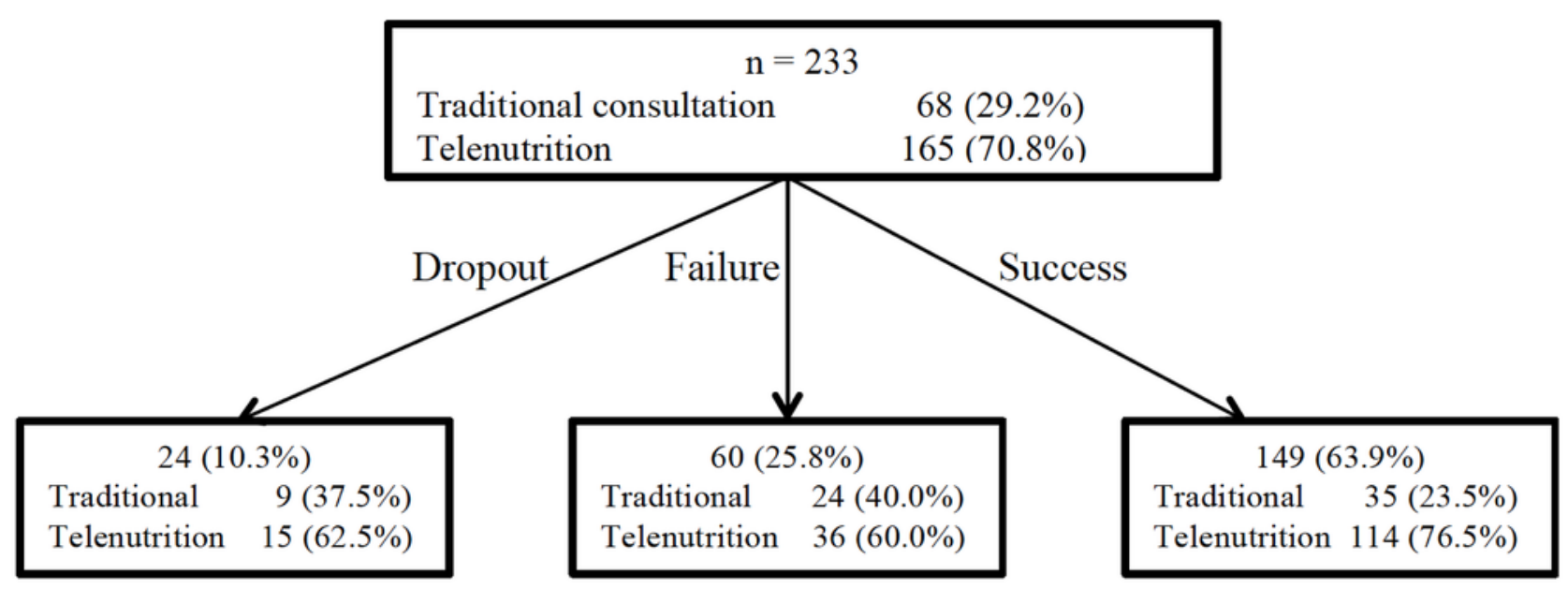

$\mathrm{Chi}^{2}$ Test: $\mathrm{p}<0.05$ 


\section{Table $\mathbf{1}$ (on next page)}

Initial values of BMI, waist, hip and waist / height index among patients who have achieved success in the traditional consultation and those who chose telenutrition. 
PeerJ Reviewing Manuscript

\begin{tabular}{|l|l|l|l|}
\hline & $\begin{array}{l}\text { Traditional } \\
\text { consultation }\end{array}$ & Telenutrition & $\begin{array}{l}\text { P (U Mann- } \\
\text { Whitney) }\end{array}$ \\
\hline Initial BMI (SD) & $27.3(4.0)$ & $27.1(4.2)$ & 0.858 \\
\hline Final BMI (SD) & $25.9(3.7)$ & $26.0(3.9)$ & 0.938 \\
\hline $\begin{array}{l}\text { Paired T test } \\
\text { iBMI vs fBMI(p) }\end{array}$ & 0.000 & 0.000 & \\
\hline Initial waist (cm) (SD) & $86.2(10.4)$ & $87.7(10.3)$ & 0.417 \\
\hline Final waist (cm) (SD) & $80.4(9.3)$ & $79.7(9.4)$ & 0.727 \\
\hline $\begin{array}{l}\text { Paired T test } \\
\text { iWaist vs fWaist (p) }\end{array}$ & 0.000 & 0.000 & \\
\hline $\begin{array}{l}\text { Initial hip (cm) (SD) } \\
\text { Final hip(cm) }\end{array}$ & $104.5(11.4)$ & $105.0(8.6)$ & 0.907 \\
\hline $\begin{array}{l}\text { Paired T test } \\
\text { iHip vs fHip (p) }\end{array}$ & $99.9(10.5)$ & $101.4(8.3)$ & 0.533 \\
\hline $\begin{array}{l}\text { iW/Initial Height index } \\
\text { (SD) }\end{array}$ & $0.55(0.06)$ & 0.000 & \\
\hline $\begin{array}{l}\text { iW/Final Height index } \\
\text { (SD) }\end{array}$ & $0.51(0.06)$ & $0.53(0.06)$ & 0.104 \\
\hline $\begin{array}{l}\text { Paired T test } \\
\text { iW/iH vs iW/fH(p) }\end{array}$ & 0.000 & $0.50(0.06)$ & 0.193 \\
\hline
\end{tabular}


Table 2 (on next page)

BMI, waist, hip and initial waist/height percentage loss in patients who have achieved success in traditional consultation and telenutrition. 
PeerJ Reviewing Manuscript

\begin{tabular}{|l|l|l|l|}
\hline & $\begin{array}{l}\text { Traditional } \\
\text { consultation }\end{array}$ & Telenutrition & $\begin{array}{l}\text { P (U Mann- } \\
\text { Whitney) }\end{array}$ \\
\hline BMI Dif \%(SD) & $1.4(1.0)$ & $1.1(1.0)$ & 0.046 \\
\hline Waist Dif \%(SD) & $5.8(3.4)$ & $5.0(3.2)$ & 0.177 \\
\hline Hip Dif \%(SD) & $4.5(2.8)$ & $3.5(3.1)$ & 0.040 \\
\hline $\begin{array}{l}\text { iWaist/Height Dif \% } \\
\text { (SD) }\end{array}$ & $0.04(0.02)$ & $0.03(0.02)$ & 0.131 \\
\hline
\end{tabular}

